\title{
Numerical characterisation of quadrics
}

\author{
Thomas Dedieu and Andreas Höring
}

\begin{abstract}
Let $X$ be a Fano manifold such that $-K_{X} \cdot C \geqslant \operatorname{dim} X$ for every rational curve $C \subset X$. We prove that $X$ is a projective space or a quadric.
\end{abstract}

\section{Introduction}

Let $X$ be a Fano manifold, that is, a complex projective manifold with ample anticanonical divisor $-K_{X}$. If the Picard number of $X$ is at least two, Mori theory shows the existence of at least two non-trivial morphisms $\varphi_{i}: X \rightarrow Y_{i}$ which encode some interesting information on the geometry of $X$. Conversely, when the Picard number equals one, Mori theory does not yield any information, and one is thus led to studying $X$ in terms of the positivity of the anticanonical bundle. A well-known example of such a characterisation is the following theorem of KobayashiOchiai.

Theorem 1.1 ([KO73]). Let $X$ be a projective manifold of dimension $n$. Suppose $-K_{X} \sim d H$, with $H$ an ample divisor on $X$. Then

(i) one has $d \leqslant n+1$ and equality holds if and only if $X \simeq \mathbb{P}^{n}$;

(ii) if $d=n$, then $X \simeq \mathbb{Q}^{n}$, where $\mathbb{Q}^{n}$ is a non-singular quadric.

The divisibility of $-K_{X}$ in the Picard group is a rather restrictive condition, so it is natural to ask for similar characterisations under (a priori) weaker assumptions. Based on Kebekus' study of singular rational curves [Keb02b], Cho, Miyaoka, and Shepherd-Barron proved a generalisation of the first part of Theorem 1.1.

Theorem 1.2 ([CMSB02, Keb02a]). Let $X$ be a Fano manifold of dimension $n$. Suppose

$$
-K_{X} \cdot C \geqslant n+1 \quad \text { for all rational curves } C \subset X \text {. }
$$

Then $X \simeq \mathbb{P}^{n}$.

The aim of this paper is to prove the following, which is a similar generalisation for the second part of Theorem 1.1.

Theorem 1.3. Let $X$ be a Fano manifold of dimension $n$. Suppose

$$
-K_{X} \cdot C \geqslant n \text { for all rational curves } C \subset X \text {. }
$$

Then $X \simeq \mathbb{P}^{n}$ or $X \simeq \mathbb{Q}^{n}$.

Received 06 September 2015, accepted in final form 17 April 2016.

2010 Mathematics Subject Classification 14J45, 14E30, 14J40, 14M22.

Keywords: rational curves, quadrics, Fano manifolds, variety of minimal rational tangents, VMRT, Mori theory. This journal is (C) Foundation Compositio Mathematica 2017. This article is distributed with Open Access under the terms of the Creative Commons Attribution Non-Commercial License, which permits non-commercial reuse, distribution, and reproduction in any medium, provided that the original work is properly cited. For commercial re-use, please contact the Foundation Compositio Mathematica.

This work was partially supported by the A.N.R. project CLASS, ANR-10-JCJC-0111. 


\section{NUMERICAL CHARACTERISATION OF QUADRICS}

This statement already appeared in a paper of Miyaoka [Miy04, Theorem 0.1], but the proof there is incomplete (cf. Remark 5.2 for instance). In this paper we borrow some ideas and tools from Miyaoka, yet give a proof based on a completely different strategy. Note also that Hwang gave a proof under the additional assumption that the general VMRT (see below) is smooth [Hwa13, Theorem 1.11], a property that does not hold for every Fano manifold [CD15, Theorem 1.10].

In the proof of Theorem 1.3, we have to assume $n \geqslant 4$; for $n \leqslant 3$ the statement follows directly from classification results.

The assumption that $X$ is Fano ensures that $\rho(X)=1$ because of the Ionescu-Wiśniewski inequality [Ion86, Theorem 0.4], [Wiś91, Theorem 1.1] (see Section 4). It is possible to remove this assumption: the Ionescu-Wiśniewski inequality together with [HN13, Theorem 1.3] enables one to deal with the case $\rho(X)>1$, and one gets the following.

Corollary 1.4. Let $X$ be a projective manifold of dimension $n$ containing a rational curve. If

$$
-K_{X} \cdot C \geqslant n \text { for all rational curves } C \subset X,
$$

then $X$ is a projective space, a hyperquadric, or a projective bundle over a curve.

(Note that under the assumptions of Corollary 1.4, if $\rho(X)=1$, then $X$ is Fano.)

Outline of the proof. In the situation of Theorem 1.3 , let $\mathcal{K}$ be a family of minimal rational curves on $X$. By Mori's bend-and-break lemma, a minimal curve $[l] \in \mathcal{K}$ satisfies $-K_{X} \cdot l \leqslant n+1$ and if equality holds, then $X \simeq \mathbb{P}^{n}$ by [CMSB02]. By our assumption we are thus left to deal with the case $-K_{X} \cdot l=n$. Then, for a general point $x \in X$ the normalisation $\mathcal{K}_{x}$ of the space parametrising curves in $\mathcal{K}$ passing through $x$ has dimension $n-2$, and by [Keb02b, Theorem 3.4] there exists a morphism

$$
\tau_{x}: \mathcal{K}_{x} \rightarrow \mathbb{P}\left(\Omega_{X, x}\right)
$$

which maps a general curve $[l] \in \mathcal{K}_{x}$ to its tangent direction $T_{l, x}^{\perp}$ at the point $x$. By [HM04, Theorem 1] this map is birational onto its image $\mathcal{V}_{x}$, the variety of minimal rational tangents (VMRT) at $x$. We denote by $\mathcal{V} \subset \mathbb{P}\left(\Omega_{X}\right)$ the total VMRT, that is, the closure of the locus covered by the VMRTs $\mathcal{V}_{x}$ for $x \in X$ general. To prove Theorem 1.3, we compute the cohomology class of the total VMRT $\mathcal{V} \subset \mathbb{P}\left(\Omega_{X}\right)$ in terms of the tautological class $\zeta$ and $\pi^{*} K_{X}$, where $\pi: \mathbb{P}\left(\Omega_{X}\right) \rightarrow X$ is the projection map. This computation is based on the construction, on the manifold $X$, of a family $\mathcal{W}^{\circ}$ of smooth rational curves such that for every $[C] \in \mathcal{W}^{\circ}$ one has

$$
\left.T_{X}\right|_{C} \simeq \mathcal{O}_{\mathbb{P}^{1}}(2)^{\oplus n} ;
$$

it lifts to a family of curves on $\mathbb{P}\left(\Omega_{X}\right)$ by associating with a curve $C \subset X$ the image $\tilde{C}$ of the morphism $C \rightarrow \mathbb{P}\left(\Omega_{X}\right)$ defined by the invertible quotient

$$
\left.\Omega_{X}\right|_{C} \rightarrow \Omega_{C} .
$$

The main technical statement of this paper is the following.

Proposition 1.5. Let $X \not \mathbb{P}^{n}$ be a Fano manifold of dimension $n \geqslant 4$, and suppose

$$
-K_{X} \cdot C \geqslant n \text { for all rational curves } C \subset X \text {. }
$$

Then, in the above notation, one has $\mathcal{V} \cdot \tilde{C}=0$ for all $[C] \in \mathcal{W}^{\circ}$.

Once we have shown this statement, a similar intersection computation involving a general minimal rational curve $l$ yields that the $\operatorname{VMRT} \mathcal{V}_{x} \subset \mathbb{P}\left(\Omega_{X, x}\right)$ is a hypersurface of degree at most 


\section{T. Dedieu AND A. Höring}

two. We then conclude with some earlier results of Araujo, Hwang, and Mok [Ara06, Hwa07, Mok08].

\section{Notation and conventions}

We work over the field $\mathbb{C}$ of complex numbers. Throughout the paper, $\mathbb{Q}^{n}$ designates a smooth quadric hypersurface in $\mathbb{P}^{n+1}$ for any positive integer $n$. Topological notions refer to the Zariski topology.

We use the modern notation for projective spaces, as introduced by Grothendieck: If $\mathcal{E}$ is a locally free sheaf on a scheme $X$, we let $\mathbb{P}(\mathcal{E})$ be $\operatorname{Proj}(\operatorname{Sym} \mathcal{E})$. If $L$ is a line in a vector space $V$, then $L^{\perp}$ designates the corresponding point in $\mathbb{P}\left(V^{\vee}\right)$. The symbols $\equiv$ and $\sim_{\mathbb{Q}}$ refer to numerical and $\mathbb{Q}$-linear equivalence, respectively.

A variety is an integral scheme of finite type over $\mathbb{C}$; a manifold is a smooth variety. A fibration is a proper surjective morphism with connected fibres $\varphi: X \rightarrow Y$ such that $X$ and $Y$ are normal and $\operatorname{dim} X>\operatorname{dim} Y>0$.

We use the standard terminology and results on rational curves, as explained in [Kol96, Chapter II], [Deb01, Chapters 2, 3, 4], and [Hwa01]. Let $X$ be a projective variety. We remind the reader that following [Kol96, II, Definition 2.11], the notation $\operatorname{RatCurves}^{\mathrm{n}}(X)$ refers to the union of the normalisations of those locally closed subsets of the Chow variety of $X$ parametrising irreducible rational curves (the superscript " $\mathrm{n}$ " is a reminder that we have normalised, and has nothing to do with the dimension).

For technical reasons, we have to consider families of rational curves on $X$ as living alternately in $\operatorname{RatCurves}^{\mathrm{n}}(X)$ and in $\operatorname{Hom}\left(\mathbb{P}^{1}, X\right)$. Our general policy is to call $\operatorname{Hom}{ }_{\mathcal{R}} \subset \operatorname{Hom}\left(\mathbb{P}^{1}, X\right)$ the family corresponding to a normal variety $\mathcal{R} \subset \operatorname{RatCurves}^{\mathrm{n}}(X)$.

\section{Preliminaries on conic bundles}

In this section, we establish some basic facts about conic bundles over a curve and compute some intersection numbers which will turn out to be crucial for the proof of Proposition 1.5. All these statements appear in one form or another in [Miy04, Section 2], but we recall them and their proofs for the clarity of exposition.

Definition 3.1. A conic bundle is an equidimensional projective fibration $\varphi: X \rightarrow Y$ such that there exist a rank three vector bundle $V \rightarrow Y$ and an embedding $X \hookrightarrow \mathbb{P}(V)$ that maps every $\varphi$-fibre $\varphi^{-1}(y)$ onto a conic (that is, the zero scheme of a degree two form) in $\mathbb{P}\left(V_{y}\right)$. The set

$$
\Delta:=\left\{y \in Y \mid \varphi^{-1}(y) \text { is not smooth }\right\}
$$

is called the discriminant locus of the conic bundle.

LEMMA 3.2. Let $S$ be a smooth surface admitting a projective fibration $\varphi: S \rightarrow T$ onto a smooth curve such that the general fibre is $\mathbb{P}^{1}$ and such that $-K_{S}$ is $\varphi$-nef. Let $F$ be a reducible $\varphi$-fibre, and suppose

$$
F=C_{1}+C_{2}+F^{\prime}
$$

where the $C_{i}$ are $(-1)$-curves and $C_{i} \not \subset \operatorname{Supp}\left(F^{\prime}\right)$. Then $F^{\prime}=\sum E_{j}$ is a reduced chain of $(-2)$ curves, and the dual graph of $F$ is as depicted in Figure 1.

Proof. Write $F^{\prime}=\sum_{j=1}^{k} a_{j} E_{j}$ with $a_{j} \in \mathbb{N}$, where $E_{1}, \ldots, E_{k}$ are the irreducible components 


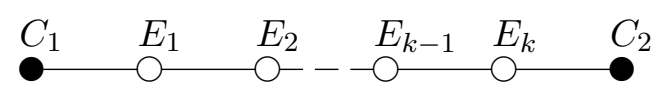

FIGURE 1.

of $F^{\prime}$. First, note that since $-K_{S} \cdot F=2$ and $-K_{S} \cdot C_{i}=1$, the fact that $-K_{S}$ is $\varphi$-nef implies $-K_{S} \cdot E_{j}=0$ for all $j$. Since $E_{j}$ is an irreducible component of a reducible fibre, we have $E_{j}^{2}<0$. Thus we see that each $E_{j}$ is a $(-2)$-curve.

We will now proceed by induction on the number of irreducible components of $F^{\prime}$, the case $F^{\prime}=0$ being trivial. Let $\mu: S \rightarrow S^{\prime}$ be the blow-down of the $(-1)$-curve $C_{2}$; then by the rigidity lemma [Deb01, Lemma 1.15], there is a morphism $\varphi^{\prime}: S^{\prime} \rightarrow T$ such that $\varphi=\varphi^{\prime} \circ \mu$. Note that $S^{\prime}$ is smooth and $-K_{S^{\prime}}$ is $\varphi^{\prime}$-nef. We also have

$$
0=C_{2} \cdot F=-1+C_{2} \cdot\left(C_{1}+\sum_{i=1}^{k} a_{i} E_{i}\right),
$$

so $C_{2}$ meets $C_{1}+\sum_{i=1}^{k} a_{i} E_{i}$ transversally in exactly one point. If $C_{2} \cdot C_{1}>0$, then $\mu_{*}\left(C_{1}\right)$ has selfintersection 0 , yet it is also an irreducible component of the reducible fibre $\mu_{*}\left(C_{1}+\sum_{i=1}^{k} a_{i} E_{i}\right)$, which gives a contradiction. Thus (up to renumbering) we can suppose $C_{2} \cdot E_{1}=1$ and $a_{1}=1$. In particular, $\mu_{*}\left(E_{1}\right)$ is a $(-1)$-curve, so

$$
\mu_{*}\left(C_{1}+\sum_{i=1}^{k} a_{i} E_{i}\right)=\mu_{*}\left(C_{1}\right)+\mu_{*}\left(E_{1}\right)+\mu_{*}\left(\sum_{i=2}^{k} a_{i} E_{i}\right)
$$

satisfies the induction hypothesis.

In the following we use that for every normal surface one can define an intersection theory using the Mumford pull-back to the minimal resolution; cf. [Sak84].

Lemma 3.3. Let $S$ be a normal surface admitting a projective fibration $\varphi: S \rightarrow T$ onto a smooth curve such that the general fibre is $\mathbb{P}^{1}$ and such that every fibre is reduced and has at most two irreducible components. Then

(i) $\varphi$ is a conic bundle;

(ii) $S$ has at most $A_{k}$-singularities; and

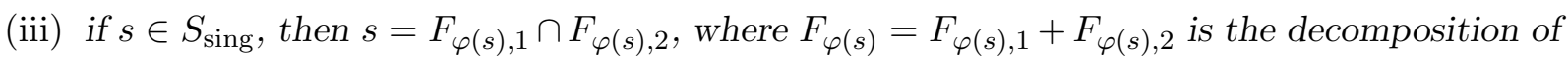
the fibre over $\varphi(s)$ in its irreducible components. In particular, $F_{\varphi(s)}$ is a reducible conic.

Proof. If a fibre $\varphi^{-1}(t)$ is irreducible, then $\varphi$ is a $\mathbb{P}^{1}$-bundle over a neighbourhood of $t$ [Kol96, II, Theorem 2.8]. Thus we only have to consider points $t \in T$ such that $S_{t}:=\varphi^{-1}(t)$ is reducible. Since $p_{a}\left(S_{t}\right)=0$ and $S_{t}=C_{1}+C_{2}$ is reduced, we see that $S_{t}$ is a union of two $\mathbb{P}^{1}$ meeting transversally in a point. Since $S_{t}=\varphi^{*} t$ is a Cartier divisor, this already implies statement (iii).

Let $\varepsilon: \hat{S} \rightarrow S$ be the canonical modification [Kol13, Theorem 1.31] of the singular points lying on $S_{t}$. Then we have

$$
K_{\hat{S}} \equiv \varepsilon^{*} K_{S}-E,
$$

with $E$ an effective $\varepsilon$-exceptional $\mathbb{Q}$-divisor whose support is equal to the $\varepsilon$-exceptional locus. Denote by $\hat{C}_{i}$ the proper transform of $C_{i}$. If $K_{\hat{S}} \cdot \hat{C}_{i}<-1$, then $\hat{C}_{i}$ deforms in $\hat{S}$ [Kol96, II, 


\section{T. Dedieu AND A. Höring}

Theorem 1.15]. Yet $\hat{C}_{i}$ is an irreducible component of a reducible $\varphi \circ \varepsilon$-fibre, so this is impossible. So we have

$$
K_{S} \cdot C_{i} \geqslant K_{\hat{S}} \cdot \hat{C}_{i} \geqslant-1
$$

for $i=1,2$. Since $K_{S} \cdot\left(C_{1}+C_{2}\right)=-2$, this implies $K_{S} \cdot C_{i}=-1$ and $E=0$. Thus $S$ has canonical singularities. Since canonical surface singularities are Gorenstein, we see that $-K_{S}$ is Cartier and $\varphi$-ample and defines an embedding

$$
S \subset \mathbb{P}\left(V:=\varphi_{*}\left(\mathcal{O}_{S}\left(-K_{S}\right)\right)\right)
$$

into a $\mathbb{P}^{2}$-bundle mapping each fibre onto a conic. This proves statement (i).

Now, let $\tilde{\varepsilon}: \tilde{S} \rightarrow S$ be the minimal resolution. It is crepant, so the divisor $-K_{\tilde{S}}$ is $\varphi \circ \tilde{\varepsilon}$-nef. Moreover, the proper transforms $\tilde{C}_{i}$ of the curves $C_{i}$ are $(-1)$-curves in $\tilde{S}$. By Lemma 3.2 this proves statement (ii).

The following fundamental lemma should be seen as an analogue of the basic fact that a projective bundle over a curve contains at most one curve with negative self-intersection.

Lemma 3.4 ([Miy04, Proposition 2.4]). Let $S$ be a normal projective surface that is a conic bundle $\varphi: S \rightarrow T$ over a smooth curve $T$, and denote by $\Delta$ the discriminant locus. Suppose that $\varphi$ has two disjoint sections $\sigma_{1}$ and $\sigma_{2}$, both contained in the smooth locus of $S$. Suppose moreover that for every $t \in \Delta$, the fibre $F_{t}$ has a decomposition $F_{t}=F_{t, 1}+F_{t, 2}$ such that

$$
\sigma_{i} \cdot F_{t, j}=\delta_{i, j}
$$

(Kronecker's delta). Assume also that we have

$$
\sigma_{1}^{2}<0 \quad \text { and } \quad \sigma_{2}^{2}<0 .
$$

Let $\varepsilon: \hat{S} \rightarrow S$ be the minimal resolution. Let $\sigma$ be a $\varphi$-section and $\hat{\sigma} \subset \hat{S}$ its proper transform. Then the following hold:

(i) If $(\hat{\sigma})^{2}<0$, then $\sigma=\sigma_{1}$ or $\sigma=\sigma_{2}$.

(ii) If $(\hat{\sigma})^{2}=0$, then $\sigma$ is disjoint from $\sigma_{1} \cup \sigma_{2}$.

Remarks 3.5. (1) In the situation above all the fibres are reduced, since there exists a section that is contained in the smooth locus.

(2) The two inequalities (3.4.2) are satisfied if there exists a birational morphism $S \rightarrow S^{\prime}$ onto a projective surface $S^{\prime}$ that contracts $\sigma_{1}$ and $\sigma_{2}$. More generally, the Hodge index theorem implies that (3.4.2) holds if there exists a nef and big divisor $H$ on $S$ such that $H \cdot \sigma_{1}=H \cdot \sigma_{2}=0$.

Proof of Lemma 3.4. Preparation: contraction to a smooth ruled surface. Lemma 3.3 applies to the surface $S$. It follows that $S$ has an $A_{k_{t}}$-singularity $\left(k_{t} \geqslant 0\right)$ in $F_{t, 1} \cap F_{t, 2}$ for every $t \in \Delta$, and no further singularities. In particular, the dual graph of $(\varphi \circ \varepsilon)^{-1}(t)$ is as described in Figure 1 for every $t \in \Delta$.

We consider the birational morphism

$$
\hat{\mu}: \hat{S} \rightarrow S^{b}
$$

defined as the composition of the blow-downs, for every $t \in \Delta$, of the proper transform $\hat{F}_{t, 1}$ of $F_{t, 1}$ and of all the $k_{t}(-2)$-curves contained in $(\varphi \circ \varepsilon)^{-1}(t)$. Since $\hat{\mu}$ is a composition of blow-downs of (-1)-curves, the surface $S^{b}$ is smooth. By the rigidity lemma [Deb01, Lemma 1.15], there is a morphism $\varphi^{b}: S^{b} \rightarrow T$. All its fibres are irreducible rational curves, so it is a $\mathbb{P}^{1}$-bundle by 


\section{NUMERICAL CHARACTERISATION OF QUADRICS}

[Kol96, II, Theorem 2.8]. Again by the rigidity lemma, $\hat{\mu}$ factors through $\varepsilon$. That is, there is a birational morphism $\mu: S \rightarrow S^{b}$ such that $\hat{\mu}=\mu \circ \varepsilon$; it is the contraction of all the curves $F_{t, 1}$ for $t \in \Delta$.

Since $\sigma_{1}$ meets $F_{t, 1}$ in a smooth point of $S$, the proper transforms $\hat{\sigma}_{1}$ and $\hat{F}_{t, 1}$ meet in the same point. Thus $\hat{\sigma}_{1}$ meets (or rather, its successive images meet) the exceptional divisor of all the blow-downs of $(-1)$-curves composing $\hat{\mu}$, and since the section $\sigma_{1}^{b}:=\hat{\mu}\left(\hat{\sigma}_{1}\right)$ is smooth, all the intersections are transversal. Vice versa we can say that $\hat{S}$ is obtained from $S^{\text {b }}$ by blowing up points on (the successive proper transforms of) $\sigma_{1}^{b}$.

By the symmetry condition (3.4.1), the curve $\sigma_{2}$ is disjoint from the $\mu$-exceptional locus, so if we set $\sigma_{2}^{b}:=\mu\left(\sigma_{2}\right)$, then we have $\left(\sigma_{2}^{b}\right)^{2}=\left(\sigma_{2}\right)^{2}<0$. Thus, in the notation of [Har77, V, Chapter 2], the surface $S^{b}$ is ruled with ruling $\varphi^{b}: S^{b} \rightarrow T$ and invariant $-e:=\left(\sigma_{2}^{b}\right)^{2}>0$. In particular, the Mori cone $\overline{\mathrm{NE}}\left(S^{b}\right)$ is generated by a general $\varphi^{b}$-fibre $F$ and $\sigma_{2}^{b}$. Since $\sigma_{1}^{b} \cdot \sigma_{2}^{b}=0$ and $\sigma_{1}^{b} \cdot F=1$, we have

$$
\sigma_{1}^{b} \equiv \sigma_{2}^{b}+e F .
$$

Conclusion. Now, let $\sigma \subset S$ be a section that is distinct from both $\sigma_{1}$ and $\sigma_{2}$. Then $\sigma^{b}:=\mu(\sigma)$ is distinct from both $\sigma_{1}^{b}$ and $\sigma_{2}^{b}$. Since $\sigma^{b} \neq \sigma_{2}^{b}$, we have

$$
\sigma^{b} \equiv \sigma_{2}^{b}+c F
$$

for some $c \geqslant e\left[\right.$ Har77, V, Proposition 2.20]. Since $\sigma^{b} \neq \sigma_{1}^{b}$, we have

$$
\sigma^{b} \cdot \sigma_{1}^{b} \geqslant \sum_{t \in \Delta} \tau_{t}
$$

where $\tau_{t}$ is the intersection multiplicity of $\sigma^{b}$ and $\sigma_{1}^{b}$ at the point $F_{t} \cap \sigma_{1}^{b}$. Denote by $\hat{\sigma} \subset \hat{S}$ the proper transform of $\sigma \subset S$, which is also the proper transform of $\sigma^{b} \subset S^{b}$. By our description of $\hat{\mu}$ as a sequence of blow-ups in $\sigma_{1}^{b}$, we obtain

$$
(\hat{\sigma})^{2}=\left(\sigma^{b}\right)^{2}-\sum_{t \in \Delta} \min \left(\tau_{t}, k_{t}+1\right) \geqslant\left(\sigma^{b}\right)^{2}-\sum_{t \in \Delta} \tau_{t}
$$

By (3.5.3) this implies

$$
(\hat{\sigma})^{2} \geqslant\left(\sigma^{b}\right)^{2}-\sigma^{b} \cdot \sigma_{1}^{b}=\sigma^{b} \cdot\left(\sigma^{b}-\sigma_{1}^{b}\right) .
$$

Plugging in (3.5.1) and (3.5.2), we obtain

$$
(\hat{\sigma})^{2} \geqslant c-e \geqslant 0
$$

This shows statement (i).

Now, suppose $(\hat{\sigma})^{2}=0$. Then by (3.5.4) we have $c=e$, hence $\sigma^{b} \cdot \sigma_{2}^{b}=0$. Being distinct, the two curves $\sigma^{b}$ and $\sigma_{2}^{b}$ are therefore disjoint, and so are their proper transforms $\hat{\sigma}$ and $\hat{\sigma}_{2}$. Now, note that $\varepsilon$ is an isomorphism in a neighbourhood of $\hat{\sigma}_{2}$, so $\sigma=\varepsilon(\hat{\sigma})$ is disjoint from $\sigma_{2}=\varepsilon\left(\hat{\sigma}_{2}\right)$. In order to see that $\sigma$ and $\sigma_{1}$ are disjoint, we repeat the same argument but contract those fibre components which meet $\sigma_{2}$. This proves statement (ii).

\section{The main construction}

4.1. Set-up. For the whole section, we let $X \not \mathbb{P}^{n}$ be a Fano manifold of dimension $n \geqslant 4$, and suppose

$$
-K_{X} \cdot C \geqslant n \text { for all rational curves } C \subset X
$$




\section{T. DEDIEU AND A. HÖRING}

this is the situation of Proposition 1.5. It then follows from the Ionescu-Wiśniewski inequality that the Picard number $\rho(X)$ equals one; see [Miy04, Lemma 4.1].

Recall that by definition, a family of minimal rational curves is an irreducible component $\mathcal{K}$ of RatCurves ${ }^{\mathrm{n}}(X)$ such that (1) the curves in $\mathcal{K}$ dominate $X$ and (2) for $x \in X$ general the algebraic set $\mathcal{K}_{x}^{b} \subset \mathcal{K}$ parametrising curves passing through $x$ is proper. We will use the following simple observation.

Lemma 4.2. In the situation of Proposition 1.5, let $l \subset X$ be a rational curve such that $-K_{X} \cdot l=n$. Then any irreducible component $\mathcal{K}$ of $\operatorname{RatCurves}^{\mathrm{n}}(X)$ containing $[l]$ is a family of minimal rational curves.

Proof. Condition (4.1.1) implies the properness of $\mathcal{K}$ [Kol96, II, (2.14)]. On the other hand, we know by [Kol96, IV, Corollary 2.6.2] that the curves parametrised by $\mathcal{K}$ dominate $X$.

4.3. Minimal rational curves and VMRTs. Since $X$ is Fano, it contains a rational curve $l$ [Mor79, Theorem 6]. Since $X \not \mathbb{P}^{n}$, there exists a rational curve with $-K_{X} \cdot l=n$ by [CMSB02], and by Lemma 4.2 there exists a family of minimal rational curves containing the point $[l] \in$ $\operatorname{RatCurves}^{\mathrm{n}}(X)$. We fix once and for all such a family, which we call $\mathcal{K}$.

For $x \in X$ general, denote by $\mathcal{K}_{x}$ the normalisation of the algebraic set $\mathcal{K}_{x}^{b} \subset \mathcal{K}$ parametrising curves passing through $x$. Every member of $\mathcal{K}_{x}^{b}$ is a free curve (this follows from the argument of [Kol96, II, proof of Theorem 3.11]), so $\mathcal{K}_{x}$ is smooth and has dimension $n-2 \geqslant 2$ [Kol96, II, (1.7) and (2.16)].

By results of Kebekus, a general curve $[l] \in \mathcal{K}_{x}^{b}$ is smooth [Keb02b, Theorem 3.3], and the tangent map

$$
\tau_{x}: \mathcal{K}_{x} \rightarrow \mathbb{P}\left(\Omega_{X, x}\right)
$$

which sends a general curve $[l]$ to its tangent direction $T_{l, x}^{\perp}$ at the point $x$, is a finite morphism [Keb02b, Theorem 3.4]. Its image $\mathcal{V}_{x}$ is called the variety of minimal rational tangents (VMRT) at $x$. The map $\tau_{x}$ is birational by [HM04, Theorem 1], so the normalisation of $\mathcal{V}_{x}$ is $\mathcal{K}_{x}$, which is smooth (this is [HM04, Corollary 1]). Also, one can associate with a general point $v \in \mathcal{V}_{x}$ a unique minimal curve $[l] \in \mathcal{K}_{x}$. We denote by $\mathcal{V} \subset \mathbb{P}\left(\Omega_{X}\right)$ the total $V M R T$, that is, the closure of the locus covered by the VMRTs $\mathcal{V}_{x}$ for $x \in X$ general. Since $\mathcal{K}_{x}$ has dimension $n-2$, the total VMRT $\mathcal{V}$ is a divisor in $\mathbb{P}\left(\Omega_{X}\right)$.

For a general $[l] \in \mathcal{K}$, one has

$$
\left.T_{X}\right|_{l} \simeq \mathcal{O}_{\mathbb{P}^{1}}(2) \oplus \mathcal{O}_{\mathbb{P}^{1}}(1)^{\oplus n-2} \oplus \mathcal{O}_{\mathbb{P}^{1}}
$$

$[$ Kol96, IV, Corollary 2.9]. We call a minimal rational curve $[l] \in \mathcal{K}$ standard if $l$ is smooth and the bundle $\left.T_{X}\right|_{l}$ has the same splitting type as in (4.3.1).

4.4. Smoothing pairs of minimal curves. For a general point $x_{1} \in X$ the curves parametrised by $\mathcal{K}_{x_{1}}$ cover a divisor $D_{x_{1}} \subset X$ [Kol96, IV, Proposition 2.5]. This divisor is ample because $\rho(X)=1$, so for $x_{2} \in X$ and $\left[l_{2}\right] \in \mathcal{K}_{x_{2}}$ the curve $l_{2}$ intersects $D_{x_{1}}$. Thus for a general point $x_{2} \in X$ we can find a chain of two standard minimal curves $l_{1} \cup l_{2}$ connecting the points $x_{1}$ and $x_{2}$. By [Kol96, II, Example 7.6.4.1] the union $l_{1} \cup l_{2}$ is dominated by a transverse union $\mathbb{P}^{1} \cup \mathbb{P}^{1}$. Since both rational curves are free, we can smooth the tree $\mathbb{P}^{1} \cup \mathbb{P}^{1}$, keeping the point $x_{1}$ fixed [Kol96, II, Theorem 7.6.1]. Since $x_{1}$ is general in $X$, this defines a family of rational curves dominating $X$; we denote by $\mathcal{W}$ the normalisation of the irreducible component of $\operatorname{Chow}(X)$ containing these rational curves. 


\section{NUMERICAL CHARACTERISATION OF QUADRICS}

4.5. Since a general member $[C]$ of the family $\mathcal{W}$ is free and $-K_{X} \cdot C=2 n$, we have $\operatorname{dim} \mathcal{W}=$ $3 n-3$. We pick an arbitrary irreducible component of the subset of $\mathcal{W}$ parametrising cycles containing $x_{1}$, and let $\mathcal{W}_{x_{1}}$ be its normalisation; then we have $\operatorname{dim} \mathcal{W}_{x_{1}}=2 n-2$. Let $\mathcal{U}_{x_{1}}$ be the normalisation of the universal family of cycles over $\mathcal{W}_{x_{1}}$. The evaluation map ev $x_{x_{1}}: \mathcal{U}_{x_{1}} \rightarrow X$ is surjective: its image is irreducible, and it contains both the divisor $D_{x_{1}}$ (because it is contained in the image of the restriction of $e_{x_{1}}$ to those members of $\mathcal{W}_{x_{1}}$ that contain a minimal curve through $x_{1}$ ) and the point $x_{2}$, which is general in $X$ (in particular, $x_{2} \notin D_{x_{1}}$ ).

Next, we choose an arbitrary irreducible component of the subset of $\mathcal{W}$ parametrising cycles passing through $x_{1}$ and $x_{2}$, and let $\mathcal{W}_{x_{1}, x_{2}}$ be its normalisation and $\mathcal{U}_{x_{1}, x_{2}}$ the normalisation of the universal family over $\mathcal{W}_{x_{1}, x_{2}}$. We denote by

$$
q: \mathcal{U}_{x_{1}, x_{2}} \rightarrow \mathcal{W}_{x_{1}, x_{2}}, \quad \text { ev }: \mathcal{U}_{x_{1}, x_{2}} \rightarrow X
$$

the natural maps. It follows from the considerations above that $\mathcal{W}_{x_{1}, x_{2}}$ is non-empty of dimension $n-1$.

By construction, a general curve $[C] \in \mathcal{W}_{x_{1}, x_{2}}$ is smooth at $x_{i}$ for $i \in\{1,2\}$, so the preimage $\mathrm{ev}^{-1}\left(x_{i}\right)$ contains a unique divisor $\sigma_{i}$ that surjects onto $\mathcal{W}_{x_{1}, x_{2}}$. Since ev is finite on the $q$-fibres and $\mathcal{W}_{x_{1}, x_{2}}$ is normal, we obtain that the degree one map $\sigma_{i} \rightarrow \mathcal{W}_{x_{1}, x_{2}}$ is an isomorphism. We call the divisors $\sigma_{i}$ the distinguished sections of $q$. We denote by $\Delta \subset \mathcal{W}_{x_{1}, x_{2}}$ the locus parametrising non-integral cycles.

Let $\operatorname{loc}_{x_{1}}^{1}$ be the locus covered by all the minimal rational curves of $X$ passing through $x_{1}$. It is itself a divisor, but may be bigger than $D_{x_{1}}$, since in general there are finitely many families of minimal curves. From now on we choose a general point $x_{2} \in X$ such that $x_{2} \notin \operatorname{loc}_{x_{1}}^{1}$ (which implies $\left.x_{1} \notin \operatorname{loc}_{x_{2}}^{1}\right)$.

Lemma 4.6. In the situation of Proposition 1.5 and using the notation introduced above, let $C$ be a non-integral cycle corresponding to a point $[C] \in \Delta$. Then $C=l_{1}+l_{2}$, with the $l_{i}$ minimal rational curves such that $x_{i} \in l_{j}$ if and only if $i=j$.

Remark. Note that we do not claim that the curves $l_{i}$ belong to the family $\mathcal{K}$. However, by construction of the family $\mathcal{W}$ as smoothings of pairs $l_{1} \cup l_{2}$ in $\mathcal{K}$, there exists an irreducible component $\Delta_{\mathcal{K}} \subset \Delta$ such that $l_{i} \in \mathcal{K}$ when $\left[l_{1}+l_{2}\right] \in \Delta_{\mathcal{K}}$.

Proof. We can write $C=\sum a_{i} l_{i}$, where the $a_{i}$ are positive integers and the $l_{i}$ are integral curves. By [Kol96, II, Proposition 2.2] all the irreducible components $l_{i}$ are rational curves. We can suppose that up to renumbering one has $x_{1} \in l_{1}$. If $a_{1} \geqslant 2$, then $-K_{X} \cdot C=2 n$, and $-K_{X} \cdot l_{1} \geqslant n$ implies $C=2 l_{1}$ and that $l_{1}$ is a minimal rational curve. Yet this contradicts the assumption $x_{2} \notin \operatorname{loc}_{x_{1}}^{1}$. Thus we have $a_{1}=1$ and since $C$ is not integral, there exists a second irreducible component $l_{2}$. Again $-K_{X} \cdot C=2 n$, and $-K_{X} \cdot l_{i} \geqslant n$ implies $C=l_{1}+l_{2}$ and that the $l_{i}$ are minimal rational curves by Lemma 4.2. The last property now follows by observing that $x_{2} \notin \operatorname{loc}_{x_{1}}^{1}$ implies $x_{1} \notin \operatorname{loc}_{x_{2}}^{1}$.

By [Kol96, II, Theorem 2.8], the fibration $q: \mathcal{U}_{x_{1}, x_{2}} \rightarrow \mathcal{W}_{x_{1}, x_{2}}$ is a $\mathbb{P}^{1}$-bundle over the open set $\mathcal{W}_{x_{1}, x_{2}} \backslash \Delta$. Although Lemma 4.6 essentially says that the singular fibres are reducible conics, it is a priori not clear that $q$ is a conic bundle (cf. Definition 3.1). This becomes true after we make a base change to a smooth curve.

Lemma 4.7. In the situation of Proposition 1.5 and using the notation introduced above, let $Z \subset$ $\mathcal{W}_{x_{1}, x_{2}}$ be a curve such that a general point of $Z$ parametrises an irreducible curve. Then there 


\section{T. DEDIEU AND A. HÖRING}

exists a finite morphism $T \rightarrow Z$ such that the normalisation $S$ of the fibre product $\mathcal{U}_{x_{1}, x_{2}} \times \mathcal{W}_{x_{1}, x_{2}} T$ has a conic bundle structure $\varphi: S \rightarrow T$ that satisfies the conditions of Lemma 3.4.

Proof. Let $\nu: \tilde{Z} \rightarrow Z$ be the normalisation, let $N$ be the normalisation of $\mathcal{U}_{x_{1}, x_{2}} \times \mathcal{W}_{x_{1}, x_{2}} \tilde{Z}$, and let $f_{N}: N \rightarrow X$ be the morphism induced by ev: $\mathcal{U}_{x_{1}, x_{2}} \rightarrow X$. Since all the curves pass through $x_{1}$ and $x_{2}$, there exist curves $Z_{1} \subset N$ and $Z_{2} \subset N$ that are contracted by $f_{N}$ onto the points $x_{1}$ and $x_{2}$, respectively. Since ev is finite on the $q$-fibres, the curves $Z_{1}$ and $Z_{2}$ are multisections of $N \rightarrow \tilde{Z}$. If $\tilde{Z}_{i}$ is the normalisation of $Z_{i}$, then the fibration $\left(N \times \tilde{Z}_{\tilde{Z}} \tilde{Z}_{i}\right) \rightarrow \tilde{Z}_{i}$ has a section given by $c \mapsto(c, c)$. Thus there exists a finite base change $T \rightarrow \tilde{Z}$ such that the normalisation $\varphi: S \rightarrow T$ of the fibre product $\left(\mathcal{U}_{x_{1}, x_{2}} \times_{\mathcal{W}_{x_{1}, x_{2}}} T\right) \rightarrow T$ has a natural morphism $f: S \rightarrow X$ induced by ev: $\mathcal{U}_{x_{1}, x_{2}} \rightarrow X$ that contracts two $\varphi$-sections $\sigma_{1}$ and $\sigma_{2}$ onto $x_{1}$ and $x_{2}$, respectively.

Since $Z \not \subset \Delta$, the general $\varphi$-fibre is $\mathbb{P}^{1}$. Moreover, by Lemma 4.6 all the $\varphi$-fibres are reduced and have at most two irreducible components. By Lemma 3.3 this implies that $\varphi$ is a conic bundle and if $s \in S_{\text {sing }}$, then $F_{\varphi(s)}$ is a reducible conic and the two irreducible components meet in $s$. Thus we have $\sigma_{i} \subset S_{\mathrm{sm}}$, where $S_{\mathrm{sm}}$ denotes the smooth locus, since otherwise both irreducible components would pass through $x_{i}$, thereby contradicting the property $x_{2} \notin \operatorname{loc}_{x_{1}}^{1}$. For the same reason we can decompose any reducible $\varphi$-fibre $F_{t}$ by defining $F_{t, i}$ as the unique component meeting the section $\sigma_{i}$. Since $\sigma_{i} \cdot F=1$ for a general $\varphi$-fibre, we see that (3.4.1) holds. Condition (3.4.2) holds with $H$ the pull-back of an ample divisor on $X$.

From this and Lemma 3.4 one deduces the following statement, in the spirit of the bend-andbreak lemma [Deb01, Proposition 3.2].

Lemma 4.8. The restriction of the evaluation map ev: $\mathcal{U}_{x_{1}, x_{2}} \rightarrow X$ to the complement of $\sigma_{1} \cup \sigma_{2}$ is quasi-finite. In particular, ev is generically finite onto its image.

Proof. We argue by contradiction. Since ev is finite on the $q$-fibres, there exists a curve $Z \subset$ $\mathcal{W}_{x_{1}, x_{2}}$ such that the natural map from the surface $q^{-1}(Z)$ onto $\operatorname{ev}\left(q^{-1}(Z)\right)$ contracts three disjoint curves $\sigma_{1}, \sigma_{2}$, and $\sigma$ onto the points $x_{1}, x_{2}$, and $x:=\operatorname{ev}(\sigma)$, respectively.

If $Z \not \subset \Delta$, then by Lemma 4.7 we can suppose, possibly up to a finite base change, that $q^{-1}(Z) \rightarrow Z$ satisfies the conditions (3.4.1) of Lemma 3.4. After a further base change we can assume that $\sigma$ is a section. Since $\sigma$ is contracted by ev, we have $\sigma^{2}<0$. By Lemma 3.4(i), this implies $\sigma=\sigma_{1}$ or $\sigma=\sigma_{2}$, which gives a contradiction.

If $Z \subset \Delta$, then all the fibres over $Z$ are unions of two minimal rational curves. Thus the normalisation of $q^{-1}(Z)$ is a union of two $\mathbb{P}^{1}$-bundles mapping onto $Z$, and by construction they contain three curves which are mapped onto points. However, a ruled surface contains at most one contractible curve, so we have a contradiction.

4.9. Since $\operatorname{dim} \mathcal{U}_{x_{1}, x_{2}}=\operatorname{dim} X$, one deduces from Lemma 4.8 that the cycles $[C] \in \mathcal{W}$ passing through $x_{1}$ and $x_{2}$ cover the manifold $X$. By [Deb01, 4.10] this implies that a general member $[C] \in \mathcal{W}_{x_{1}, x_{2}}$ is a 2 -free rational curve [Deb01, Definition 4.5]. Since $-K_{X} \cdot C=2 n$, this forces

$$
f^{*} T_{X} \simeq \mathcal{O}_{\mathbb{P}^{1}}(2)^{\oplus n},
$$

where $f: \mathbb{P}^{1} \rightarrow C \subset X$ is the normalisation of $C$. As a consequence, one sees from [Kol96, II, Theorem 3.14.3] that a general member $[C] \in \mathcal{W}$ is a smooth rational curve in $X$.

Let $\operatorname{Hom}_{\mathcal{W}}^{\circ} \subset \operatorname{Hom}\left(\mathbb{P}^{1}, X\right)$ be the irreducible open set parametrising morphisms $f: \mathbb{P}^{1} \rightarrow X$ such that the image $C:=f\left(\mathbb{P}^{1}\right)$ is smooth, the associated cycle $[C] \in \operatorname{Chow}(X)$ is a point in $\mathcal{W}$, 


\section{NUMERICAL CHARACTERISATION OF QUADRICS}

and $f^{*} T_{X}$ has splitting type (4.9.1). By what precedes, the image of $\operatorname{Hom}_{\mathcal{W}}^{\circ}$ in $\mathcal{W}$ under the natural map $\operatorname{Hom}\left(\mathbb{P}^{1}, X\right) \rightarrow \operatorname{Chow}(X)$ is a dense open set $\mathcal{W}^{\circ} \subset \mathcal{W}$.

4.10. Denote by $\pi: \mathbb{P}\left(\Omega_{X}\right) \rightarrow X$ the projection map. We define an injective map

$$
i: \operatorname{Hom}_{\mathcal{W}}^{\circ} \hookrightarrow \operatorname{Hom}\left(\mathbb{P}^{1}, \mathbb{P}\left(\Omega_{X}\right)\right)
$$

by sending $f: \mathbb{P}^{1} \rightarrow X$ to the morphism $\tilde{f}: \mathbb{P}^{1} \rightarrow \mathbb{P}\left(\Omega_{X}\right)$ corresponding to the invertible quotient $f^{*} \Omega_{X} \rightarrow \Omega_{\mathbb{P}^{1}}$. For $[C] \in \mathcal{W}^{\circ}$ with normalisation $f$, we call $[\tilde{C}]$ the member of $\operatorname{Chow}\left(\mathbb{P}\left(\Omega_{X}\right)\right)$ corresponding to the lifting $\tilde{f}$.

We let $\operatorname{Hom}_{\mathcal{W}}$ be the image of $i$. Note that it parametrises a family of rational curves that dominates $\mathbb{P}\left(\Omega_{X}\right)$, but it is not an irreducible component of $\operatorname{Hom}\left(\mathbb{P}^{1}, \mathbb{P}\left(\Omega_{X}\right)\right)$. Indeed, $\operatorname{Hom}_{\mathcal{W}}$ is contained in a (much bigger) irreducible component defined by morphisms corresponding to arbitrary quotients $f^{*} \Omega_{X} \rightarrow \mathcal{O}_{\mathbb{P}^{1}}(-2)$.

The following property is well known to experts. Since Hom $\tilde{\mathcal{W}}$ is not an open subset of the space $\operatorname{Hom}\left(\mathbb{P}^{1}, \mathbb{P}\left(\Omega_{X}\right)\right)$, we have to adapt the proof of [Kol96, II, Proposition 3.7].

Lemma 4.11. In the situation of Proposition 1.5, let $\mathcal{V}_{0} \subset \mathcal{V}$ be a dense, Zariski-open set in the total VMRT $\mathcal{V}$, and let $\tilde{C}:=\tilde{f}\left(\mathbb{P}^{1}\right)$ be a rational curve parametrised by a general point of $\operatorname{Hom}_{\mathcal{W}}$. Then one has

$$
(\mathcal{V} \cap \tilde{C}) \subset\left(\mathcal{V}_{0} \cap \tilde{C}\right) .
$$

Proof. Set $Z:=\mathcal{V} \backslash \mathcal{V}_{0}$. A point $z \in \mathbb{P}\left(\Omega_{X}\right)$ is of the form $z=\left(v_{z}^{\perp}, x\right)$, where $\mathbb{C} v_{z} \subset T_{X, x}$ is a tangent direction in $X$ at $x=\pi(z)$. So for all $p \in \mathbb{P}^{1}$ and $z=\left(v_{z}^{\perp}, x\right) \in \mathbb{P}\left(\Omega_{X}\right)$, the morphisms $[\tilde{f}] \in \operatorname{Hom}_{\mathcal{W}}$ mapping $p$ to $z$ correspond to morphisms $f: \mathbb{P}^{1} \rightarrow X$ in $\operatorname{Hom}_{\mathcal{W}}^{\circ}$ mapping $p$ to $x$ with tangent direction $\mathbb{C} v_{z}$. Since $f$ has splitting type (4.9.1), the set of these morphisms has dimension exactly $n$. It follows that

$$
\operatorname{Hom}_{\mathcal{W}, Z}:=\left\{[\tilde{f}] \in \operatorname{Hom}_{\mathcal{W}} \mid \tilde{f}\left(\mathbb{P}^{1}\right) \cap Z \neq \emptyset\right\}=\bigcup_{z \in Z} \bigcup_{p \in \mathbb{P}^{1}}\left\{[\tilde{f}] \in \operatorname{Hom}_{\mathcal{W}} \mid \tilde{f}(p)=z\right\}
$$

has dimension at most $\operatorname{dim} Z+1+n$.

Now $\mathcal{V} \subset \mathbb{P}\left(\Omega_{X}\right)$ is a divisor, and $Z$ has codimension at least one in $\mathcal{V}$, so $Z$ has dimension at most $2 n-3$, and the set $\operatorname{Hom}_{\mathcal{W}, Z}$ above has dimension at most $3 n-2$. Since $\operatorname{Hom}_{\mathcal{W}}^{\circ}$ has dimension $3 n$ and $\operatorname{Hom}_{\mathcal{W}}^{\circ} \rightarrow \operatorname{Hom}_{\mathcal{W}}$ is injective, a general point $[\tilde{f}] \in \operatorname{Hom}_{\mathcal{W}}$ is not in $\operatorname{Hom}_{\mathcal{W}, Z}$.

We need one more technical statement.

Lemma 4.12. In the situation of Proposition 1.5 and using the notation introduced above, let $[f] \in \operatorname{Hom}_{\mathcal{W}}^{\circ}$ be a general point. Then for every $x \in f\left(\mathbb{P}^{1}\right)$ we have $f\left(\mathbb{P}^{1}\right) \not \subset \operatorname{loc}_{x}^{1}$.

Proof. Fix two general points $x_{1}, x_{2} \in X$. A general morphism $[f] \in \operatorname{Hom}_{\mathcal{W}}^{\circ}$ passing through $x_{1}$ and $x_{2}$ is 2 -free, and up to reparametrisation we have $f(0)=x_{1}$ and $f(\infty)=x_{2}$. Set $g:=\left.f\right|_{\{0, \infty\}}$; then $f$ is free over $g$ [Kol96, II, Definition 3.1]. Now, suppose that such a curve has the property $f\left(\mathbb{P}^{1}\right) \subset \operatorname{loc}_{x_{0}}^{1}$ for some $x_{0} \in f\left(\mathbb{P}^{1}\right)$. Thus $x_{1}, x_{2} \in \operatorname{loc}_{x_{0}}^{1}$, hence by symmetry $x_{0} \in\left(\operatorname{loc}_{x_{1}}^{1} \cap \operatorname{loc}_{x_{2}}^{1}\right)$. Yet the intersection

$$
\operatorname{loc}_{x_{1}}^{1} \cap \operatorname{loc}_{x_{2}}^{1}
$$

has codimension two in $X$. By [Kol96, II, Proposition 3.7] a general deformation of $f$ over $g$ is disjoint from this set. 


\section{T. DEDIEU AND A. HÖRING}

4.13. Proof of Proposition 1.5. Arguing by contradiction, we suppose $\mathcal{V} \cdot \tilde{C}>0(\tilde{C}$ is not contained in $\mathcal{V}$ for general $\left.[C] \in \mathcal{W}^{\circ}\right)$. Applying Lemma 4.11 with

$$
\mathcal{V}_{0}:=\left\{v^{\perp} \in \mathcal{V} \mid \mathbb{C} v=T_{l, \pi(v)}, \text { where }[l] \in \mathcal{K} \text { is standard }\right\},
$$

we see that for a general point $[C] \in \mathcal{W}$ there exist a point $x_{1} \in C$ and a standard curve $[l] \in \mathcal{K}_{x_{1}}$ such that

$$
T_{C, x_{1}}=T_{l, x_{1}} .
$$

We shall now reformulate the property (4.13.1) in terms of the universal family $\mathcal{U}_{x_{1}, x_{2}}$, with $x_{2}$ a point chosen in $C \backslash \operatorname{loc}_{x_{1}}^{1}$ thanks to Lemma 4.12. Consider the blow-up $\varepsilon: \tilde{X} \rightarrow X$ at the point $x_{1}$, with exceptional divisor $E_{1}$. There is a rational map ev : $\mathcal{U}_{x_{1}, x_{2}} \rightarrow \tilde{X}$ such that $\varepsilon \circ \tilde{\text { ev }}=\mathrm{ev}$ (on the locus where ev is defined); since the general member of $\mathcal{W}_{x_{1}, x_{2}}$ is smooth at $x_{1}$, this map ev is well defined in a general point of $\sigma_{1}$ and restricts to a rational map $\sigma_{1} \rightarrow E_{1}$. The latter is dominant and therefore generically finite, because the general member of $\mathcal{W}_{x_{1}, x_{2}}$ is 2 -free. In particular, we may assume that it is finite in a neighbourhood of the point $C \cap \sigma_{1}$.

We then consider the proper transform $\tilde{l}$ of $l$ under $\varepsilon$, and let $\Gamma$ be an irreducible component of $\tilde{\mathrm{ev}}^{-1}(\tilde{l})$ passing through $C \cap \sigma_{1}$. It is a curve that is mapped to a curve in $\mathcal{W}_{x_{1}, x_{2}}$ by $q$. Applying the same construction to the divisor $D_{x_{1}} \subset X$, one gets a prime divisor $G \subset \mathcal{U}_{x_{1}, x_{2}}$ mapped surjectively onto $D_{x_{1}}$ and $\mathcal{W}_{x_{1}, x_{2}}$ by ev and $q$, respectively.

In general the curve $\Gamma$ could be contained in the locus where $\left.q\right|_{G}$ or ev $\left.\right|_{G}$ is not étale. However, the standard rational curves $[l] \in \mathcal{K}$ such that a corresponding curve $\Gamma$ is not contained in these ramification loci form a non-empty Zariski-open set in $\mathcal{K}$. Hence their tangent directions define a non-empty Zariski open set in $\mathcal{V}$. Applying Lemma 4.11 a second time, we can thus replace $C$ by a general curve $C^{\prime}$ such that $\left[C^{\prime}\right] \in \mathcal{W}^{\circ} \cap \mathcal{W}_{x_{1}, x_{2}}$ and hence $l$ by a general $\left[l^{\prime}\right] \in \mathcal{K}_{x_{1}}$ such that there exists a curve $\Gamma^{\prime} \subset G$ such that $q\left(\Gamma^{\prime}\right)$ is a curve, ev $\left(\Gamma^{\prime}\right)=l^{\prime}$, and both maps $\left.q\right|_{G}$ and ev $\left.\right|_{G}$ are étale at the general point $x \in \Gamma^{\prime}$. By construction the point $C^{\prime} \cap \sigma_{1}$ lies on $\Gamma^{\prime}$. This gives a contradiction to Proposition 4.14 below.

Proposition 4.14 ([Miy04, Lemma 3.9]). In the situation of Proposition 1.5, let $x_{1}, x_{2} \in X$ be general points and $[l]$ a general member of $\mathcal{K}_{x_{1}}$. Consider an irreducible curve $\Gamma \subset \mathcal{U}_{x_{1}, x_{2}}$ such that $\operatorname{ev}(\Gamma)=l$ and $q(\Gamma)$ is a curve, and assume that there exists a prime divisor $G \subset \mathcal{U}_{x_{1}, x_{2}}$ mapped onto $D_{x_{1}}$ by ev and containing $\Gamma$, such that both maps $\left.q\right|_{G}$ and $\left.\mathrm{ev}\right|_{G}$ are étale at a general point of $\Gamma$. Then $\Gamma \cap \sigma_{1}$ does not contain any point $C \cap \sigma_{1}$ with $[C] \in \mathcal{W}^{\circ} \cap \mathcal{W}_{x_{1}, x_{2}}$.

We give the proof for the sake of completeness.

Proof. Since $[l]$ is general in $\mathcal{K}_{x_{1}}$, we have

$$
\left.T_{X}\right|_{l} \simeq \mathcal{O}_{\mathbb{P}^{1}}(2) \oplus \mathcal{O}_{\mathbb{P}^{1}}(1)^{n-2} \oplus \mathcal{O}_{\mathbb{P}^{1}}
$$

and $\mathcal{K}_{x_{1}}$ is smooth with tangent space $H^{0}\left(l, N_{l / X}^{+} \otimes \mathcal{O}_{l}\left(-x_{1}\right)\right)$ at $[l]$, where $\mathcal{E}^{+}$denotes the ample part of a vector bundle $\mathcal{E} \rightarrow \mathbb{P}^{1}$, that is, its ample subbundle of maximal rank.

Let $x \in \Gamma$ be a general point, and set $y=\mathrm{ev}(x) \in l$. For some analytic neighbourhood $V \subset \mathcal{K}_{x_{1}}$ of $[l]$, we have an evaluation map

$$
\mathrm{ev}_{x_{1}}: \mathbb{P}^{1} \times V \longrightarrow D_{x_{1}}
$$

which is étale at $(y,[l])$, and the tangent space to $D_{x_{1}}$ at $y$ is thus

$$
T_{D_{x_{1}}, y}=T_{l, y} \oplus\left(N_{l / X}^{+} \otimes \mathcal{O}_{l}\left(-x_{1}\right)\right)_{y}=\left.T_{X}\right|_{l, y} ^{+} .
$$




\section{NUMERICAL CHARACTERISATION OF QUADRICS}

Since ev $\left.\right|_{G}$ is étale in $x$, we obtain that the tangent map

$$
d_{x} \mathrm{ev}: T_{\mathcal{U}_{x_{1}, x_{2}}, x} \rightarrow \mathrm{ev}^{*}\left(T_{X, \mathrm{ev}(x)}\right)
$$

maps $T_{G, x}$ isomorphically into the ample part; that is, we have

$$
d_{x} \mathrm{ev}\left(T_{G, x}\right) \simeq \mathrm{ev}^{*}\left(\left.T_{X}\right|_{l, \mathrm{ev}(x)} ^{+}\right) .
$$

We argue by contradiction and suppose that there exists a $[C] \in \mathcal{W}^{\circ} \cap \mathcal{W}_{x_{1}, x_{2}}$ such that $\left(C \cap \sigma_{1}\right) \in\left(\Gamma \cap \sigma_{1}\right)$. Since $\Gamma$ maps onto $l$, it is not contained in the divisor $\sigma_{1}$. Since the smooth rational curve $C$ is 2 -free, there exists by semicontinuity a neighbourhood $U$ of $[C] \in \mathcal{W}_{x_{1}, x_{2}}$ parametrising 2 -free smooth rational curves. For a 2 -free rational curve, the evaluation morphism ev is smooth in the complement of the distinguished divisors $\sigma_{i}$ [Kol96, II, Proposition 3.5.1]. Thus if we denote by $R \subset \mathcal{U}_{x_{1}, x_{2}}$ the ramification divisor of ev, then $\sigma_{1}$ is the unique irreducible component of $R$ containing the point $C \cap \sigma_{1}$. Thus $\Gamma$ is not contained in the ramification divisor of ev.

Since $q(\Gamma)$ is a curve, there exists by Lemma 4.7 a finite base change $T \rightarrow q(\Gamma)$, with $T$ a smooth curve, such that the normalisation $S$ of the fibre product $T \times \mathcal{W}_{x_{1}, x_{2}} \mathcal{U}_{x_{1}, x_{2}}$ is a surface with a conic bundle structure $\varphi: S \rightarrow T$ satisfying the conditions of Lemma 3.4. After a further base change we may suppose that there exists a $\varphi$-section $\Gamma_{1}$ that maps onto $\Gamma$. Note that since we obtained $S$ by a base change from $\mathcal{U}_{x_{1}, x_{2}}$, the ramification divisor of the map $\mu: S \rightarrow \mathcal{U}_{x_{1}, x_{2}}$ is contained in the $\varphi$-fibres; that is, its image by $\varphi$ has dimension zero. In particular, $\Gamma_{1}$ is not contained in this ramification locus.

Since the rational curve $C$ is smooth and 2 -free, the universal family $\mathcal{U}_{x_{1}, x_{2}}$ is smooth in a neighbourhood of $C \cap \sigma_{1}$. Thus $\sigma_{1}$ is a Cartier divisor in a neighbourhood of $C \cap \sigma_{1}$, and we can use the projection formula to see that

$$
\Gamma_{1} \cdot \mu^{*} \sigma_{1}=\mu_{*}\left(\Gamma_{1}\right) \cdot \sigma_{1}>0 .
$$

In particular, $\Gamma_{1}$ is not disjoint from the distinguished sections in the conic bundle $S \rightarrow T$. Now, let $\varepsilon: \hat{S} \rightarrow S$ be the minimal resolution of singularities and $\hat{\Gamma}_{1}$ the proper transform of $\Gamma_{1}$. Since the distinguished sections are in the smooth locus of $S$, the section $\hat{\Gamma}_{1}$ is not disjoint from the distinguished sections of $\hat{S} \rightarrow T$. We shall now show

$$
\left(\hat{\Gamma}_{1}\right)^{2} \leqslant 0
$$

which gives a contradiction to Lemma 3.4.

Denote by $f: \hat{\Gamma}_{1} \rightarrow l$ the restriction of ev $\circ \mu \circ \varepsilon: \hat{S} \rightarrow X$. Since $\hat{\Gamma}_{1}$ is not in the ramification locus of $\mu \circ \varepsilon$ and $\Gamma$ is not in the ramification divisor of ev, the tangent map

$$
\left.\left.T_{\hat{S}}\right|_{\hat{\Gamma}_{1}} \rightarrow f^{*} T_{X}\right|_{l}
$$

is generically injective. Since $\hat{\Gamma}_{1}$ is a $(\varphi \circ \varepsilon)$-section, we have an isomorphism

$$
\left.T_{\hat{S} / T}\right|_{\hat{\Gamma}_{1}} \simeq N_{\hat{\Gamma}_{1} / \hat{S}}
$$

Since $l$ has the standard splitting type (4.3.1), we have a (unique) trivial quotient $\left.f^{*} T_{X}\right|_{l} \rightarrow \mathcal{O}_{\hat{\Gamma}_{1}}$, and thanks to (4.14.2) we are done if we prove that the natural map

$$
\left.\left.\left.T_{\hat{S} / T}\right|_{\hat{\Gamma}_{1}} \hookrightarrow T_{\hat{S}}\right|_{\hat{\Gamma}_{1}} \rightarrow f^{*} T_{X}\right|_{l} \rightarrow \mathcal{O}_{\hat{\Gamma}_{1}}
$$

is not zero. It is sufficient to check this property for a general point in $\hat{\Gamma}_{1}$, and since $\hat{\Gamma}_{1} \rightarrow \Gamma$ is generically étale, it is sufficient to check that for a general $x \in \Gamma$, the natural map

$$
T_{\mathcal{U}_{x_{1}, x_{2}} / \mathcal{W}_{x_{1}, x_{2}, x}} \rightarrow \operatorname{ev}^{*}\left(T_{X, \mathrm{ev}(x)}\right)
$$




\section{T. DEDIEU AND A. HÖRING}

does not have its image in the ample part $\mathrm{ev}^{*}\left(\left.T_{X}\right|_{l, \mathrm{ev}(x)} ^{+}\right)$. Yet if $T_{\mathcal{U}_{x_{1}, x_{2}} / \mathcal{W}_{x_{1}, x_{2}}, x}$ maps into the ample part, the decomposition $T_{\mathcal{U}_{x_{1}, x_{2}}, x}=T_{\mathcal{U}_{x_{1}, x_{2}} / \mathcal{W}_{x_{1}, x_{2}, x}} \oplus T_{G, x}$ (given by the fact that $\left.q\right|_{G}$ is étale in $x$ ) combined with (4.14.1) implies that the tangent map

$$
d_{x} \mathrm{ev}: T_{\mathcal{U}_{x_{1}, x_{2}}, x} \rightarrow \mathrm{ev}^{*}\left(T_{X, \mathrm{ev}(x)}\right)
$$

cannot be surjective. Since $\Gamma$ is not contained in the ramification locus of ev, this is impossible.

\section{Proof of the main theorem}

5.1. Proof of Theorem 1.3. If $X \simeq \mathbb{P}^{n}$, we are done, so suppose that this is not the case. Consider the family of minimal rational curves $\mathcal{K}$ constructed in Section 4 and the associated total $\operatorname{VMRT} \mathcal{V}$. Denote by $d \in \mathbb{N}$ the degree of a general $\operatorname{VMRT} \mathcal{V}_{x} \subset \mathbb{P}\left(\Omega_{X, x}\right)$.

Step 1: Using the family $\mathcal{W}^{\circ}$. In this step we prove

$$
\mathcal{V} \sim_{\mathbb{Q}} d\left(\zeta-\frac{1}{n} \pi^{*} K_{X}\right),
$$

where $\zeta$ is the tautological divisor class on $\mathbb{P}\left(\Omega_{X}\right)$. Note that $\mathbb{P}\left(\Omega_{X}\right)$ has Picard number two, so we can always write

$$
\mathcal{V} \sim_{\mathbb{Q}} a \zeta+b \frac{-1}{n} \pi^{*} K_{X}
$$

with $a, b \in \mathbb{Q}$. Now, let $\mathcal{W}^{\circ}$ be the family of rational curves constructed in Section 4 , and let $\tilde{C}$ be the lifting of a curve $C \in \mathcal{W}^{\circ}$. By Proposition 1.5 we have $\mathcal{V} \cdot \tilde{C}=0$. Since by the definition of $\tilde{C}$ one has $\zeta \cdot \tilde{C}=-2$ and $-\frac{1}{n} \pi^{*} K_{X} \cdot \tilde{C}=2$, it follows that $a=b$. Since $\mathcal{V}_{x}=\left.\left.\mathcal{V}\right|_{\mathbb{P}\left(\Omega_{X, x}\right)} \sim_{\mathbb{Q}} d \zeta\right|_{\mathbb{P}\left(\Omega_{X, x}\right)}$, we have $a=b=d$. This proves (5.1.1).

Step 2: Bounding the degree $d$. Denote by $\mathcal{K}^{\circ} \subset \mathcal{K}$ the open set parametrising smooth standard rational curves in $\mathcal{K}$. We define an injective map

$$
j: \mathcal{K}^{\circ} \hookrightarrow \operatorname{RatCurves}^{\mathrm{n}}\left(\mathbb{P}\left(\Omega_{X}\right)\right)
$$

by mapping a curve $l$ to the image $\tilde{l}$ of the morphism $s: l \rightarrow \mathbb{P}\left(\Omega_{X}\right)$ defined by the invertible quotient $\left.\Omega_{X}\right|_{l} \rightarrow \Omega_{l}$. We denote by $\tilde{\mathcal{K}}^{\circ}$ the image of $j$. Let us start by showing that $\tilde{\mathcal{K}}^{\circ}$ is dense in an irreducible component of RatCurves ${ }^{\mathrm{n}}\left(\mathbb{P}\left(\Omega_{X}\right)\right)$. Since $l$ is standard, the relative Euler sequence restricted to $\tilde{l}$ implies $H^{0}\left(\tilde{l},\left.T_{\mathbb{P}\left(\Omega_{X}\right) / X}\right|_{\tilde{l}}\right)=0$. Then, using the exact sequence

$$
\left.\left.\left.\left.0 \rightarrow T_{\mathbb{P}\left(\Omega_{X}\right) / X}\right|_{\tilde{l}} \rightarrow T_{\mathbb{P}\left(\Omega_{X}\right)}\right|_{\tilde{l}} \rightarrow\left(\pi^{*} T_{X}\right)\right|_{\tilde{l}} \simeq T_{X}\right|_{l} \rightarrow 0,
$$

we obtain that the Zariski tangent space of $\operatorname{Hom}\left(\mathbb{P}^{1}, \mathbb{P}\left(\Omega_{X}\right)\right)$ at a point corresponding to the rational curve $\tilde{l}$ has dimension at most $h^{0}\left(l,\left.T_{X}\right|_{l}\right)=2 n$. Thus we can use [Kol96, II, Theorem 2.15] to see that RatCurves ${ }^{\mathrm{n}}\left(\mathbb{P}\left(\Omega_{X}\right)\right)$ has dimension at most $2 n-3$ at the point $[\tilde{l}]$, which is exactly the dimension of $\tilde{\mathcal{K}}^{\circ}$.

By construction the lifted curves $\tilde{l}$ are contained in $\mathcal{V}$. Consequently, the open set $\tilde{\mathcal{K}}_{0} \subset$ $\operatorname{RatCurves}^{\mathrm{n}}\left(\mathbb{P}\left(\Omega_{X}\right)\right)$ is actually an open set in $\operatorname{RatCurves}^{\mathrm{n}}(\mathcal{V})$. Since $\mathcal{V} \subset \mathbb{P}\left(\Omega_{X}\right)$ is a hypersurface, the algebraic set $\mathcal{V}$ has locally complete intersection singularities. Thus we can apply [Kol96, II, Theorems 1.3 and 2.15] and obtain

$$
2 n-3=\operatorname{dim} \tilde{\mathcal{K}}_{0} \geqslant\left.\operatorname{deg} \omega_{\mathcal{V}}^{-1}\right|_{\tilde{l}}+(2 n-2)-3 .
$$

We thus have $\left.\operatorname{deg} \omega_{\mathcal{V}}^{-1}\right|_{\tilde{l}} \leqslant 2$. 


\section{NUMERICAL CHARACTERISATION OF QUADRICS}

Now, by construction we have $-\frac{1}{n} \pi^{*} K_{X} \cdot \tilde{l}=1$ and $\zeta \cdot \tilde{l}=-2$. Since $K_{\mathbb{P}\left(\Omega_{X}\right)}=2 \pi^{*} K_{X}-n \zeta$, the adjunction formula and (5.1.1) yield

$$
2 \geqslant\left.\operatorname{deg} \omega_{\mathcal{V}}^{-1}\right|_{\tilde{l}}=-\left(K_{\mathbb{P}\left(\Omega_{X}\right)}+\mathcal{V}\right) \cdot \tilde{l}=d .
$$

Step 3: Conclusion. If $d=1$ or $d=2$ but $\mathcal{V}_{x}$ is reducible, we obtain a contradiction to [Hwa07, Theorem 1.5] (cf. also [Ara06, Theorem 3.1]). If $d=2$ and $\mathcal{V}_{x}$ is irreducible, $\mathcal{V}_{x}$ is normal [Har77, II, Example 6.5(a)] and therefore isomorphic to its normalisation $\mathcal{K}_{x}$, which is smooth (see Section 4). It is thus a smooth quadric and we conclude by [Mok08, Main Theorem].

Remark 5.2. Let us explain the difference between our proof and Miyaoka's approach. In the notation of Section 4, Miyaoka considers the family $\mathcal{W}_{x_{1}, x_{2}}$. As we have seen above the evaluation map ev: $\mathcal{U}_{x_{1}, x_{2}} \rightarrow X$ is generically finite; his goal is to prove that ev is birational. He therefore analyses the preimage $\mathrm{ev}^{-1}\left(l_{1} \cup l_{2}\right)$, where $l_{1}, l_{2} \subset X$ are general minimal curves passing through $x_{1}$ and $x_{2}$, respectively, such that $\left[l_{1} \cup l_{2}\right] \in \mathcal{W}_{x_{1}, x_{2}}$. If $\Gamma \subset \mathrm{ev}^{-1}\left(l_{1} \cup l_{2}\right)$ is an irreducible curve mapping onto $l_{1}$, one can make a case distinction: If $q(\Gamma)$ is a curve that is not contained in the discriminant locus $\Delta \subset \mathcal{W}_{x_{1}, x_{2}}$ (Case $\mathbf{C}$ in [Miy04, division into cases before Lemma 3.8, p. 227]), Miyaoka makes a very interesting observation, which we stated as Proposition 4.14. However, the analysis of the 'trivial' case (loc. cit., Case $\mathbf{A}$ ), where $q(\Gamma)$ is a point, is not correct: it is not clear that $q(\Gamma)=\left[l_{1} \cup l_{2}\right]$, because there might be another curve in $\mathcal{W}_{x_{1}, x_{2}}$ which is of the form $l_{1} \cup l_{2}^{\prime}$ with $l_{2} \neq l_{2}^{\prime}$. This possibility is an obvious obstruction to the birationality of ev and invalidates [Miy04, Corollaries 3.11(2) and 3.13(1)]. The following example shows that this possibility does indeed occur.

ExAmple 5.3. Let $H \subset \mathbb{P}^{n}$ be a hyperplane and $A \subset H \subset \mathbb{P}^{n}$ a projective manifold of dimension $n-2$ and degree $a$ with $3 \leqslant a \leqslant n$. Let $\mu: X \rightarrow \mathbb{P}^{n}$ be the blow-up of $\mathbb{P}^{n}$ along $A$. Then $X$ is a Fano manifold [Miy04, Remark 4.2] and $-K_{X} \cdot C \geqslant n$ for every rational curve $C \subset X$ passing through a general point (the $\mu$-fibres are, however, rational curves with $-K_{X} \cdot C=1$ ). The general member of a family of minimal rational curves $\mathcal{K}$ is the proper transform of a line that intersects $A$. Consider the family $\mathcal{W}$ whose general member is the strict transform of a reduced, connected degree two curve $C$ such that $A \cap C$ is a finite scheme of length two. For general points $x_{1}, x_{2} \in X$ the (normalised) universal family $\mathcal{U}_{x_{1}, x_{2}} \rightarrow \mathcal{W}_{x_{1}, x_{2}}$ is a conic bundle and the evaluation map ev: $\mathcal{U}_{x_{1}, x_{2}} \rightarrow X$ is generically finite. We claim that ev is not birational.

Proof of the claim. For simplicity of notation we also denote by $x_{1}$ and $x_{2}$ the corresponding points in $\mathbb{P}^{n}$. Let $l_{1} \subset \mathbb{P}^{n}$ be a general line through $x_{1}$ that intersects $A$. Since $x_{2} \in \mathbb{P}^{n}$ is general, there exists a unique plane $\Pi$ containing $l_{1}$ and $x_{2}$. Moreover, the intersection $\Pi \cap A$ consists of exactly a points, one of them the point $A \cap l_{1}$. For every point $x \in \Pi \cap A$ other than $A \cap l_{1}$, there exists a unique line $l_{2, x}$ through $x$ and $x_{2}$. By Bezout's theorem $l_{1} \cup l_{2}$ is connected, so its proper transform belongs to $\mathcal{W}_{x_{1}, x_{2}}$. Yet this shows that $\mathrm{ev}^{-1}\left(l_{1}\right)$ contains $a-1>1$ copies of $l_{1}$, one for each point $x \in \Pi \cap A \backslash l_{1} \cap A$. This proves the claim.

Let us conclude this example by mentioning that the conic bundle $\mathcal{U}_{x_{1}, x_{2}} \rightarrow \mathcal{W}_{x_{1}, x_{2}}$ does not satisfy the symmetry conditions of Lemma 3.4 .

\section{ACKNOWLEDGEMENTS}

We warmly thank Stéphane Druel for his numerous comments during this project. We also thank the anonymous referee for the careful reading and useful remarks. 


\section{T. DEDIEU AND A. HÖRING}

\section{REFERENCES}

Ara06 C. Araujo, Rational curves of minimal degree and characterizations of projective spaces, Math. Ann. 335 (2006), no. 4, 937-951; https://doi.org/10.1007/s00208-006-0775-2.

CD15 C. Casagrande and S. Druel, Locally unsplit families of rational curves of large anticanonical degree on Fano manifolds, Int. Math. Res. Not. 2015 (2015), no. 21, 10756-10800; https: //doi.org/10.1093/imrn/rnv011.

CMSB02 K. Cho, Y. Miyaoka, and N.I. Shepherd-Barron, Characterizations of projective space and applications to complex symplectic manifolds, Higher Dimensional Birational Geometry (Kyoto, 1997), Adv. Stud. Pure Math., vol. 35 (Math. Soc. Japan, Tokyo, 2002), 1-88.

Deb01 O. Debarre, Higher-dimensional algebraic geometry, Universitext (Springer-Verlag, New York, 2001); https://doi.org/10.1007/978-1-4757-5406-3.

Har77 R. Hartshorne, Algebraic geometry, Grad. Texts in Math., vol. 52 (Springer-Verlag, New York Heidelberg, 1977); https://doi.org/10.1007/978-1-4757-3849-0.

HM04 J.-M. Hwang and N. Mok, Birationality of the tangent map for minimal rational curves, Asian J. Math. 8 (2004), no. 1, 51-63; https://doi.org/10.4310/AJM.2004.v8.n1.a6.

HN13 A. Höring and C. Novelli, Mori contractions of maximal length, Publ. Res. Inst. Math. Sci. 49 (2013), no. 1, 215-228; https://doi.org/10.4171/PRIMS/103.

Hwa01 J.-M. Hwang, Geometry of minimal rational curves on Fano manifolds, School on Vanishing Theorems and Effective Results in Algebraic Geometry (Trieste, 2000), ICTP Lect. Notes, vol. 6 (Abdus Salam Int. Cent. Theoret. Phys., Trieste, 2001), 335-393.

Hwa07_, Deformation of holomorphic maps onto Fano manifolds of second and fourth Betti numbers 1, Ann. Inst. Fourier (Grenoble) 57 (2007), no. 3, 815-823; https://doi.org/10. 5802/aif. 2278.

Hwa13 U Varieties of minimal rational tangents of codimension 1, Ann. Sci. Éc. Norm. Supér. (4) 46 (2013), no. 4, 629-649.

Ion86 P. Ionescu, Generalized adjunction and applications, Math. Proc. Cambridge Philos. Soc. 99 (1986), no. 3, 457-472; https://doi.org/10.1017/S0305004100064409.

Keb02a S. Kebekus, Characterizing the projective space after Cho, Miyaoka and Shepherd-Barron, Complex Geometry (Göttingen, 2000) (Springer, Berlin, 2002), 147-155; https://doi.org/10. 1007/978-3-642-56202-0_10.

Keb02b , Families of singular rational curves, J. Algebraic Geom. 11 (2002), no. 2, 245-256; https://doi.org/10.1090/S1056-3911-01-00308-3.

KO73 S. Kobayashi and T. Ochiai, Characterizations of complex projective spaces and hyperquadrics, J. Math. Kyoto Univ. 13 (1973), 31-47; http://projecteuclid.org/euclid.kjm/ 1250523432.

Kol96 J. Kollár, Rational curves on algebraic varieties, Ergeb. Math. Grenzgeb. (3), vol. 32 (SpringerVerlag, Berlin, 1996); https://doi.org/10.1007/978-3-662-03276-3.

Kol13 S Singularities of the minimal model program, Cambridge Tracts in Math., vol. 200 (Cambridge Univ. Press, Cambridge, 2013); https://doi.org/10.1017/CB09781139547895.

Miy04 Y. Miyaoka, Numerical characterisations of hyperquadrics, Complex Analysis in Several Variables-Memorial Conference of Kiyoshi Oka's Centennial Birthday, Adv. Stud. Pure Math., vol. 42 (Math. Soc. Japan, Tokyo, 2004), 209-235.

Mok08 N. Mok, Recognizing certain rational homogeneous manifolds of Picard number 1 from their varieties of minimal rational tangents, Third International Congress of Chinese Mathematicians. Part 1, 2, AMS/IP Stud. Adv. Math., 42, pt. 1, vol. 2 (Amer. Math. Soc., Providence, RI, 2008), 41-61.

Mor79 S. Mori, Projective manifolds with ample tangent bundles, Ann. of Math. 110 (1979), no. 3, 593-606; https://doi.org/10.2307/1971241. 


\section{NUMERICAL CHARACTERISATION OF QUADRICS}

Sak84 F. Sakai, Weil divisors on normal surfaces, Duke Math. J. 51 (1984), no. 4, 877-887; https: //doi.org/10.1215/S0012-7094-84-05138-X.

Wiś91 J.A. Wiśniewski, On contractions of extremal rays of Fano manifolds, J. reine angew. Math. 417 (1991), 141-157; https://doi.org/10.1515/crll.1991.417.141.

Thomas Dedieu thomas.dedieu@m4x.org

Institut de Mathématiques de Toulouse (CNRS UMR 5219), Université Paul Sabatier, 31062 Toulouse Cedex 9, France

Andreas Höring hoering@unice.fr

Laboratoire de Mathématiques J. A. Dieudonné, UMR 7351 CNRS, Université de Nice SophiaAntipolis, 06108 Nice Cedex 02, France 